\title{
Collective memory based on semantic annotation among economic intelligence actors
}

\author{
Bensattalah Aissa* \\ National High School for Computer Science (E.S.I), \\ 16000 Oued-Smar, Algiers, Algeria \\ and \\ National Institute of Technology, \\ BP 78 Zaaroura, 14000 Tiaret, Algeria \\ E-mail: a_bensattalah@esi.dz \\ *Corresponding author
}

\section{Fahima Nader and Rachid Chalal}

National High School for Computer Science (E.S.I), 16000 Oued-Smar, Algiers, Algeria

E-mail: f_nader@esi.dz

E-mail: r_chalal@esi.dz

\begin{abstract}
Many enterprises are reflected on strategies and tools that facilitate the knowledge sharing and exploiting the collective intelligence among their actors. Economic intelligence actors collaborate to solve a decisional problem, they use significant mental effort, and so they share a common knowledge that can indicate to other actors directions to follow or avoid. Indeed, whenever an actor explores knowledge or a relevant document, it enriches the collective knowledge of the memory via annotations. To ensure this collaboration in solving a decisional problem among actors in a context of economic intelligence, in this article we propose a conceptual model using ontologies to represent collaborative semantic annotations between economic intelligence actors in order to capitalise on and reuse the knowledge shared in a collective memory.
\end{abstract}

Keywords: economic intelligence; semantic annotation; collective memory; ontology; modelling interaction meaning; knowledge capitalisation; knowledge reuse; collective intelligence management; collaborative problem solving.

Reference to this paper should be made as follows: Aissa, B., Nader, F. and Rachid, C. (2014) 'Collective memory based on semantic annotation among economic intelligence actors', Int. J. Collaborative Intelligence, Vol. 1, No. 1, pp.18-32.

Biographical notes: Bensattalah Aissa is a Lecturer at the Ibn Khaldoun University, Tiaret, Algeria. He is a PhD student in the National Higher School of Computer Science, ESI, Algiers, Algeria. His research focuses on models and annotation tools for collective memory between the decision makers in a context of competitive intelligence. His research interests include information systems and business intelligence, ontology, semantic annotation, and collective memory. 
Fahima Nader is a Researcher/Lecturer in the Laboratory of Systems Design Methods (LMCS) at the National Computer Science Engineering School, ESI, Algiers, Algeria, where she teaches undergraduate and graduate computer science courses. She earned her PhD degree in the same school in 2008. Her research focuses on models and annotation tools for collective memory between the decision maker and hurdy-gurdy in a context of business intelligence. Her research interests include information systems and business intelligence, service oriented architecture, decision support systems, and collective memory.

Rachid Chalal is a Professor at the National School of Computer Science (ESI ex. INI) since 1994. He is responsible for a team of researchers in the field of decision support at the Laboratory of LMCS ESI. His main research concerns cover information systems, competitive intelligence, and decision support.

\section{Introduction}

The need for strategic tools is becoming increasingly important because knowledge handled, the information needs and information is often exchanged in electronic format (internet, intranet, newspapers, note synthesis, mail, letter, database, report, articles, theses, laws and decrees). Knowledge and/or relevant information are a critical resource for an enterprise, economic intelligence (EI) actors. According to Li et al. (2010), every enterprise has data, information or knowledge, special knowledge play an important role in decision making process, there are many information sources, and the web has also become a common and huge information source. According to Oliveira et al. (2004), the internet is becoming the basic infrastructure for communication among people and companies. Its main services (web and e-mail system) offer a large and growing text collection containing a huge volume of useful information. According to Deng and Luo (2007) the new applications of Web 2.0 (Blog, Wiki, SNS, and RSS) provide new opportunities and channels which make the gathering of information a very important task in the strategic management and decision-making in enterprises.

The transformations generated by the knowledge economy, the changing environment, the complexities of decisional problems and failure in finding relevant information in the mass data, today management of collective intelligence is an important focus in the enterprise that requires a strategy for managing collective mental effort (memorising, re-use). These mental efforts are generally the time spent for interpretation of tacit knowledge or seeking of informational needs during the reading of a document for the decision making.

In our work, we consider that document management and content management become important for the EI actors, because it handles a large number of activities on the documents while working. The annotation is a current activity made by the EI actors on their documents.

In this paper, we propose a collective memory to the EI actors. This memory is built using the documents and the annotations used by the actors to annotate these documents. These annotations often specify specific activities that EI actors want to do on these documents. In this work, we first begin by explaining concepts related to EI and the need for a collective memory for the actors. We describe later the communication ontology [its 
design process (Noy et al., 2001) and the resulted ontology]. Finally, we present the tool which is used by the EI actors to build its collective memory.

\section{Economic intelligence}

Information and knowledge has become an important resource for decision making process or solving decisional problems. The main purpose of EI is to help people to solve decisional problems or to find relevant information for decision making. Several tools are available as data mining, web mining, text mining, annotation tools, web service, data warehouse, decision support system (DSS) or smart decision support system (IDSS).

In Said and Bretones (2009), EI shows a major focus on explicit knowledge, these EI processes encompass various fields ranging from information search to knowledge analysis and interpretation. It also covers information sharing, collaborative work. EI aims to increase the organisation's absorptive capacity and innovation systems, decision making and understanding. In addition to that (Thiery and David, 2002) EI is a process that embodies decision making. Decision marking can be regarded as an outcome of mental processes (cognitive process) leading to the selection of an action course among several alternatives. According to Li et al. (2010), decision making process or problem solving begin with defining the problem. All problems can be expressed as information, and there must exist knowledge which helps to solve the problem. According to Thiery and David (2002), and Olusoji et al. (2010), EI process is made up of the following stages:

1 identification of decisional problems

2 transformation of decisional problem into information search problem

3 identification of relevant information sources.

4 collection of relevant information.

5 processing the collected information for the extract of indicators for the decision

6 interpretation of indicators.

7 decision making.

All the EI phases involve actors who collaborate to produce actionable knowledge for decision making purpose; it implies that EI process requires high level of collaboration among its actors to find relevant information for solving decisional problems (Odumuyiwa and David, 2008). According to Philippe (2009), EI is an informational process used in order to solve decisional problem or making decision. According to Philippe (2009), EI approach is a means of resolving decision problems based on information gathering and the interpretation given by the EI actors on the extracted information from available heterogeneous sources, when various actors work together in a collaborative environment, they need to coordinate their interactions. Managing interactions, among collaborating EI actors, is an important element to provide effective knowledge production and sharing. 
There are two major EI actors involved in the process (Thiery and David, 2002; Olusoji et al., 2010):

Decision maker who must formulate an exact description of the decision-problem; watcher (person whose function is to obtain relevant problem associated with the decision problem at hand) who must locate, supervise; validate and emphasise the strategic information needed for solving the problem.

To solve the problem of communicational flow among EI actors Philippe (2009) proposed three actions to trace, annotate and memorise to establish the collaboration confidence of the watcher and the decision maker (i.e., EI actors), and other authors (Odumuyiwa and David, 2008) proposed a communication model for knowledge sharing in collaborative information retrieval among EI actors. Olusoji et al. (2010) proposed a model for managing interactions among collaborating EI actors for dynamic knowledge capitalisation using annotation.

The objective of this paper is to capture the interactions between the EI actors during decisional problem solving (interaction, communication, collaboration and coordination) through the use of semantic annotations in order to create a collective memory which facilitates the exchange of knowledge and saves these annotations in order to re-use them to make decision. This is illustrated in Figure 1.

Figure 1 Collaboration between economic intelligence actors to solve a decisional problem (see online version for colours)

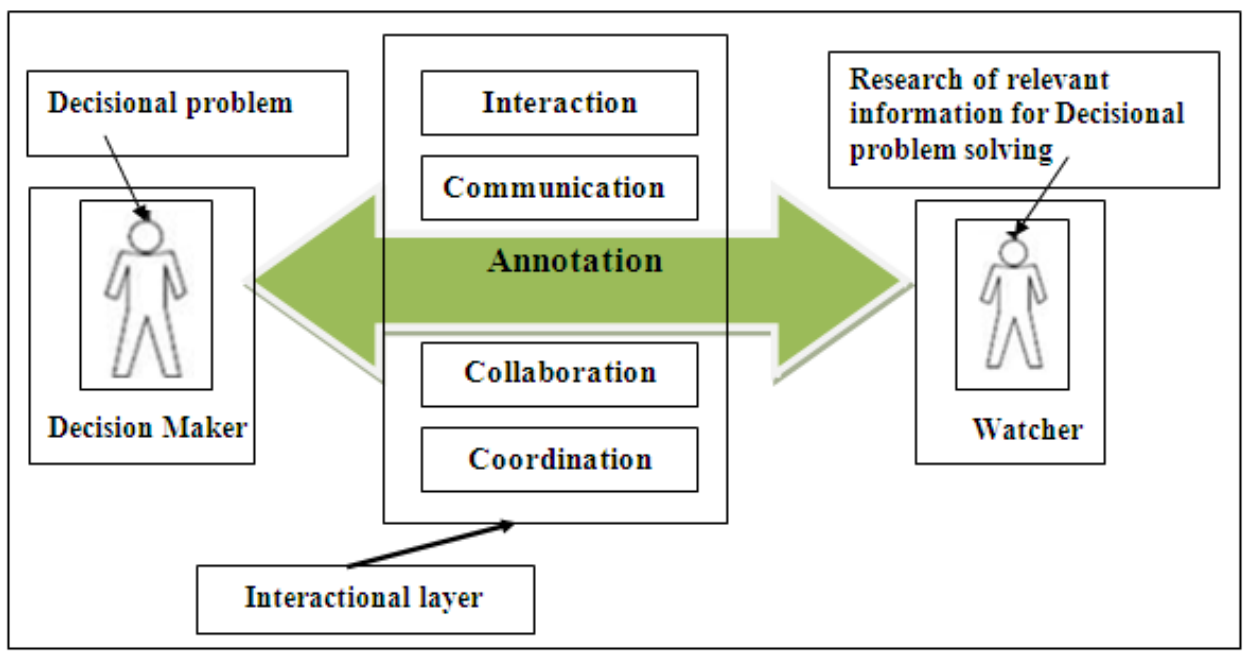

\section{Modelling interaction meaning}

Collaborative problem solving process requires a structure of communication between the EI actors. In this context, we model interaction meaning (see Figure 2) on the document by semantic annotation. Interaction meaning is the text annotated on the document linked to a concept of communication ontology for represent semantic annotation. 
Figure 2 Modelling interaction meaning (see online version for colours)

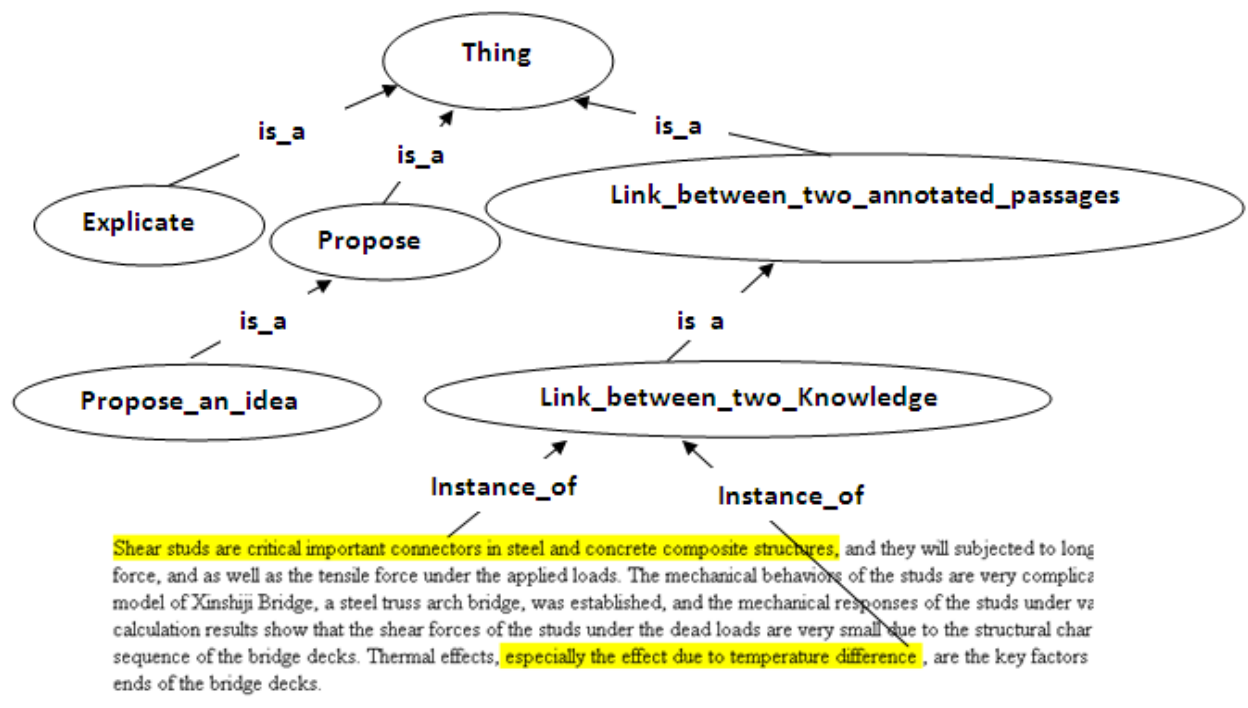

\section{Collective memory}

In Dixon et al. (1997), collaborative problem solving occurs in situations in which two or more individuals cooperate in appraising, representing, and solving a variety of cognitive tasks. Ardakani and Oloonabadi (2011) define a collective memory which represents a series of events collectively remembered by a group of people who share it and involve themselves in shaping it. This is an element that can be used as a driver to achieve these objectives. In David et al. (2005), the collective memory concept can also be specialised to a group level and used to support work groups engaged in repetitive decision-making activities, the advantages of capturing collective memory are many, including simplification of the process, codification of decision strategies, carryover of knowledge when group composition changes, etc. According to Paul et al. (2004), the use of collective memory information provides the study participants focused attention on the cognitive-conflict task domain and leads to faster decision-making. In the work of Cabanac et al. (2007), the authors proposed expertise memory. That is why they introduced the 'decisional annotation' concept to allow analysts to annotate tables of multidimensional databases, to share comments between coworkers, and to foster opinion exchange within discussion threads. Moreover, the resulting annotations and discussions are stored in a shared expertise memory in order to keep a record of the decision making process.

In this work, the collective memory has a significant importance for EI actors; they need to be assisted by computer tools 'collective memory' to better manage their collective knowledge, reflection, collect the mental effort spent for information or result interpretation. 
The need for EI tool is becoming increasingly important with the emergence of information exchange in digital form and collaborative for solve the complexity of decisional problem. The advantages of this collective memory are:

To memorise the knowledge generated by the interactions around solving decisional problem, to memorise and to re-use the efforts mental of group decision makers and to make the global comprehension of decisional problem and its strategy to solve.

\section{Ontology-based model for annotation semantics}

The annotation is a mental effort applied to a document in the reading activity during the research and analysis information, the reasons from which the annotations made are the interpretation of information, to memorise knowledge, traceability. According to Boudebza et al. (2013), annotation represents the externalised knowledge which reflects personal knowledge and experiences of the annotator. According to Philippe (2009), the annotation represents the vector of the reusability of the informational elements of the watcher, Barber et al. (2005) intend to reduce document reading time by the collective annotation, in the work of Cabanac et al. (2007) collective annotation is used to allow discussing and debating on the other hand the annotation proposed in the work of Azouaou and Desmoulins (2006) aims to memorise, to re-use and share knowledge.

The purpose of this paper is to model the activity made on the document by the economic intelligence actors in the phase of problem-solving in an ontology model in order to use it to annotate. Ontology can be useful for representing the concepts and their instances. In our ontology these concepts are activities made on the document. According to Moscato et al. (2009), ontology-based annotation is one of the leading trends in the creation of metadata. It uses ontologies as vocabularies. In Macario and Medeiros (2009), proposed a framework that tackles interoperability problems via semantic annotations, which are based on multiple ontologies in order to efficient information retrieval for planning and decision-making in domain of geospatial data for agriculture. Azouaou and Desmoulins (2006) proposed a model of semantic annotations suitable for teachers by using ontologies to represent teachers' annotations. The model is well adapted to teachers' activities with regard to both their pedagogical and domain expertise. Wahiba and Nouha (2011) presented an approach based on semantic annotation of resumes for e-recruitment process can be useful for many human resource applications: retrieval, classification, extraction of element dependencies, analysis of relationships between elements, etc. Perez-Gallardo et al. (2013) proposed recommender systems to implement collective intelligence, which capitalises the knowledge of human collectives. The recommendations are intended to provide interesting elements to users. Recommender systems are used collectively tagged by the users' community with a semantic sense. And in the work of Boudebza et al. (2013) proposed approach is based contextual semantic annotation model, for improving knowledge reusability within online communities of practice of e-learning (CoPEs). 


\section{Construction of communication ontology}

EI actors use a specific shared community language to annotate. This language is a set of common annotation objectives for solving these problems. The annotation's objective is considered as a central point among EI actors, because it defines the semantics of the annotation.

The EI actors collaborate to solve a decisional problem. Thus, they share a common knowledge which makes it possible to indicate, to other actors, directions to be followed or to avoid. In order to take into account the semantic annotations of the relations in documents which express the reasons why actors communicate author of a decisional problem (proposal, clarification, dependent between knowledge, traced knowledge...), who are identified automatically by an engine of annotation.

In addition, in this article we use protégé tool to conduct the communication ontology. There are several methods for the development of ontology. To design the communication ontology, we follow the iterative method, for the development of ontology, proposed by Noy et al. (2001).

The field and scope of the ontology: we begin the development of the ontology by defining its domain and its scope using these questions: what is the area that will cover the ontology? The domain ontology is the different activities that are performed by EI actors on the documents. What are the goals of development of the ontology? The ontology is designed in order to formalise and clarify the semantics of annotations produced by economic intelligence actors on the documents.

Reusing existing ontology: We can reuse all or some of these ontologies after they suit our needs. Azouaou and Desmoulins (2006) proposes a formalisation of the teacher's annotation objectives. These objectives are not adapted to the context of EI. We adapted some concepts in our ontology. Baker (2009) proposed concepts to facilitate the communication structure for example (explanation, proposal, etc.). Identification and structuring of the ontology concepts: To identify the main concepts of the ontology. We analysed the process of communication and the various objectives of an annotation between actors in EI context at the time collaboration to solve a problem.

Basic concepts are used by the EI actors as a way to organise the explanation interactional and to facilitate communicational flows to solve decisional problem in a collaborative context. In addition, and how linked enters various knowledge found the mass significant of the web documents, how exploiting and memorising of the individual efforts mental and collective (cognitive process of the actors) used in the course of the solving decisional problem for the decision-making. These concepts are structured using a shared ontology we detail below.

Actors, for example, can bind between several knowledge (passages annotated) which they find in different documents or the same document, to consign another actor to consult a document, a reference or a significant annotated passage, binds between two similar or contradictory proposals, binds a concept ambiguous by one or more knowledge to explain it, etc. These concepts give the direction for which the EI actors solve a decisional problem and monitor dynamically changing information which is needed as soon as its formalism until a its solving and thus all the interactions carried out by the actors. Once annotated with these activities categories, the documents are then indexed and become easily retrieved and searched by the actors.

We have organised and structure these activities interaction in the following ontology 'Communication ontology' as shows in Figure 3. 
Figure 3 Concepts of communication ontology are developed using protégé editor (see online version for colours)

\begin{tabular}{|c|c|}
\hline owl:Thing & Link_between_two_annotated_passages \\
\hline A Annotation & Link_between_two_decisional_problems \\
\hline Annatator & $\boldsymbol{\nabla}$ Link_between_two_decisional_problems_similar \\
\hline Creation_date_annotation & Notion_similar \\
\hline Document_uri & some_similar_part \\
\hline Id_annotation & Methode_of_resolving_similar \\
\hline$\nabla$ Explicate & Link_between_two_Knowledges \\
\hline Explicate_a_concept & Link_between_two_concepts \\
\hline Explicate_a_quetion & Link_between_two_ideas \\
\hline Explicate_a_reasrch_problem_information & - Link_between_two_documents \\
\hline Explicate_an_answer & Link_between_two_proposals \\
\hline Explicate_an_idea & Link_between_two_clarification \\
\hline$\nabla$ Propose & Link_between_two_decisions \\
\hline Propose_a_solution & Link_decisional_problem_with_problem_of_search_information \\
\hline Propose_an_approch_to_information_retrevail & Link_problem_of_search_information_with_relevart_information \\
\hline Propose_an_idea & $\nabla$ Synthesis_of_annotations \\
\hline$\nabla$ To_consign & $\nabla$ Comparaison_between_two_annotations \\
\hline Methode_to_be_followed & Same_context \\
\hline Reference_to_be_seen & Different_context \\
\hline$\nabla$ A_decision & Similar_meaning \\
\hline Agreed_to_the_decision & Contradictory_meaning \\
\hline Not_agree_with_the_decision & $\boldsymbol{\nabla}$ To_memorize \\
\hline & To_memorize_an_informational_need \\
\hline & To_memorize_an_objective_to_be_waited \\
\hline & To_memorize_a_solution \\
\hline & To_memorize_a_task_to_come \\
\hline
\end{tabular}

Some instances of communication ontology concepts are added automatically in the base of annotation. For example, the concept 'Creation date annotation' is Creation date of annotation and the 'annotator' concept is personal information about the annotator (actors).

\section{Illustration of the use of the collective memory}

We illustrate below the use of collective memory application following two examples where actors annotated their documents using mental activities on document taken from the communication ontology. We chose two concepts for illustration, the concept of 'link between two annotated passages' and the concept of 'Contradictory meaning'.

We represent the passage annotated by $\mathrm{Ai}$, the document read by $\mathrm{Di}$, the creation date of the annotation by $\mathrm{Ti}$, concepts by $\mathrm{Ci}$ and actors or annotators by USERi.

Example 1: being a research information need to understand a concept in an ambiguous decision problem. The USER1 after collecting documents and during the reading, he found three passages annotated to explain the concept ambiguous. To memorise these three passages (the contents of the texts found in the documents) immediately, he uses the annotation as follow: 
- One moment $\mathrm{T} 1$ the concept $\mathrm{C} 1$ bound by the passage annotated $\mathrm{A} 1$ that it found in the D1 document.

- One T2 moment the concept (C1) bound by a second passage annotated A2 that it found in the same D1 document.

- One moment $\mathrm{T} 3$ the $\mathrm{C} 1$ concept bound by a third passage annotated $\mathrm{A} 3$ that it found in the document $\mathrm{D} 2$, etc.

- The annotated passages A1, A2 and A3 are bound by the concept of ontology bound between two annotated passages.

Example 2: A second USER2 read the annotations which make by USER1 and after an analysis (mental effort), it found that A1 and A2, two passages annotated, are in contradiction with the explanation of ambiguous $\mathrm{C} 1$, it bound these two passages by the concept of ontology 'Contradictory meaning' in the moment T4, so it is memorised them and the USER1 is informed.

The uses of the concepts of annotations syntheses are very significant to create a new knowledge or to make a decision.

\section{Collective memory architecture using semantic web technologies}

Collective memory architecture is based on the Web 2.0 concepts. Is to exploit the Web 2.0 concept on collaborative to solve problem decisional through annotation. Global architecture is divided into two parts: the client and the server side (see Figure 4).

- Client side: The user interface is a web browser. It is developed using a JavaScript and document object model (DOM).

- Server side: The application in the server part are developed using the PHP, XML, AJAX technology and are deployed in EasyPHP server.

The annotations are stored in the XML (Extensible Markup Language) format (see Figure 7), which is a semantic web language. Ontologies are represented in OWL DL (see Figure 5) language for automatic processing of the document annotation and Reasoner with using Pellet OWL, SPARQL query can be used to query an OWL model to filter out annotations with specific characteristics. We use jQuery is a JavaScript library that focuses on the interaction between JavaScript (including Ajax) and HTML, to simplify common commands for JavaScript. And DOM technology is a W3C standard to access and modify the style and structure of the document for annotation. The XMLHttpRequest object to perform queries on the server. We use Asynchronous JavaScript and XML (AJAX) for communication between the server and the client. 
Figure 4 Collective memory architecture (see online version for colours)

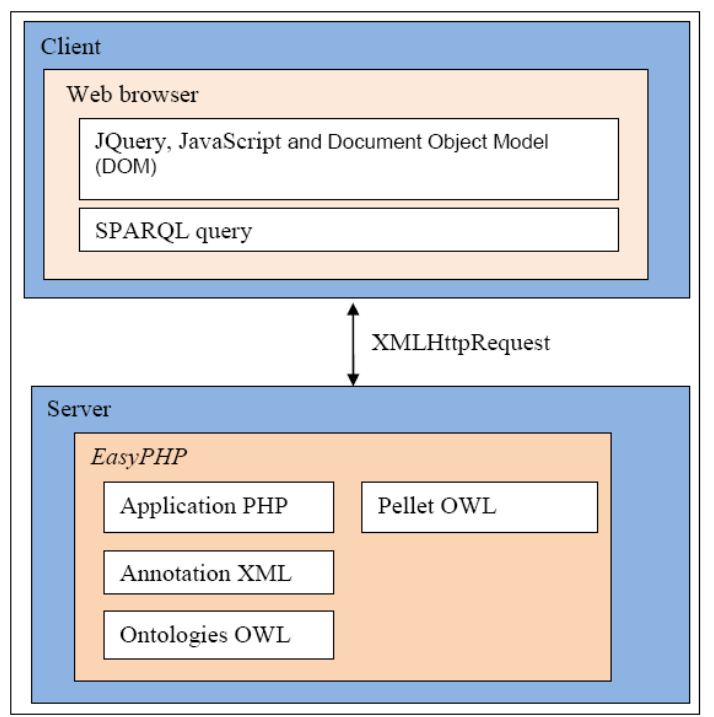

Figure 5 Part of communication ontology using language OWL (see online version for colours)

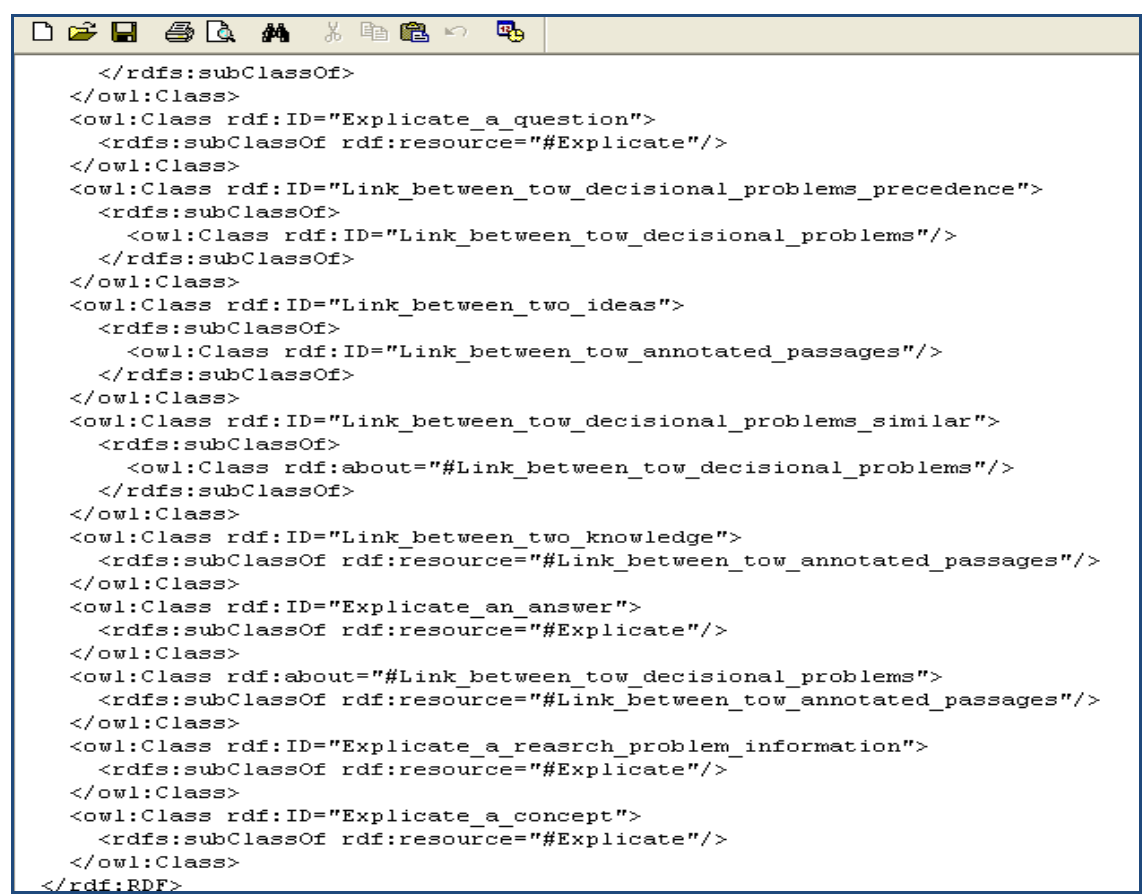

The following scenario (see Figure 6) explains the interaction between the user and the collective memory tool at the creation of a new annotation. 
- the user selects passages of a text in the document

- then, the user chooses the colour of the annotation

- the browser propose new window, where the user choose the objective of the annotation that represents in ontology (see Figure 3), for example, 'Link between ideas'

- the user saves the annotation.

These annotations save in server with XML format (see Figure 7). XML format contains three important tags, annotation colour in tag $<$ colr $>$, annotated text in tag $<$ val $>$, annotation objective from ontology in tag $<$ titre $>$.

Figure 6 Example of scenario for explain using communication ontology for semantic annotation (see online version for colours)

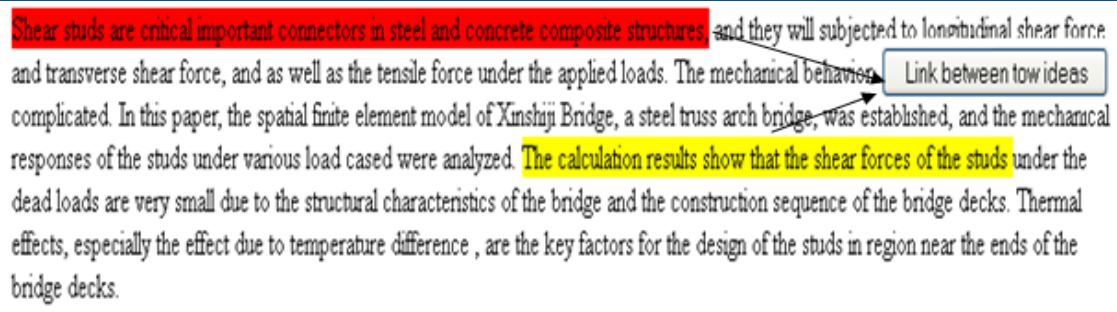

Figure 7 XML format for save annotations (see online version for colours)

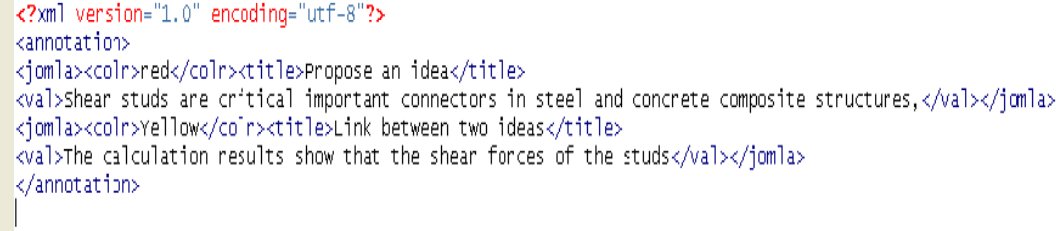

\section{Implementation}

The collective memory tool has been developed using the semantic web technologies and it allows the actors to make semantic annotation on their documents. The following Figure 8 represents the global interface of our tool used by EI actors to handle their documents, annotated them according to the concepts chosen from the ontology in the part 2, share the decisional problem in the part1 and the opening of the documents in part 3. For example, the decision maker shares the decisional problem "the decision maker want to know the influence of the stud on the mechanical behaviour of structure" in the part 1 and an actor of EI bounds two passages annotated after a reading and an analysis by the objective of annotation chosen from the ontology and yellow colour 'Contradictory meaning', these two passages annotated are shared by another actor which wants to understand a concept ambiguous in the decisional problem 'Stud' annotated by the red colour. 
Figure 8 Collective memory interface (see online version for colours)

Ling
Link between tow knowledges
Link between tow ideas
Link between tow concepts
Explicate a question
Propose
Synthesis of annotations
Comparison between two annotations
Same context
Different context
$\quad$ Similar meaning
Contradictory meaning
To consign
Reference to be seen
Method to be followed

\section{Semantic search}

In collective memory tool, the actors can use the semantic search, which exploits the ontologies used to describe the annotations.

For example, the actor can select in the ontology the concept 'Contradictory meaning' (see Figure 9), then collective memory tool displays the annotations that have the objective attribute $=$ 'Contradictory meaning'.

Another example:

Figure 9 Semantic search based on ontology interface (see online version for colours)

\begin{tabular}{|l|}
\hline Semantic search \\
Explicate \\
Explicate a concept \\
Explicate an idea \\
Explicate a research problem information \\
Explicate an answer \\
Explicate a question \\
Propose \\
Synthesis of annotations \\
Comparison between two annotations \\
Same context \\
Different context \\
Similar meaning \\
Contradictory meaning \\
\hline
\end{tabular}


Actors during their information search and analysis document to solve a problem they need to link between two or more knowledge found in a document or in different documents. When the actor wants to display all the knowledge memorising during the search, the actors select in the ontology the concept 'Link between two knowledge' (see Figure 10), then collective memory tool displays the annotations that have the objective attribute $=$ 'Link between two knowledge'.

Figure 10 Example of semantic search for all the knowledge memorising during the search (see online version for colours)

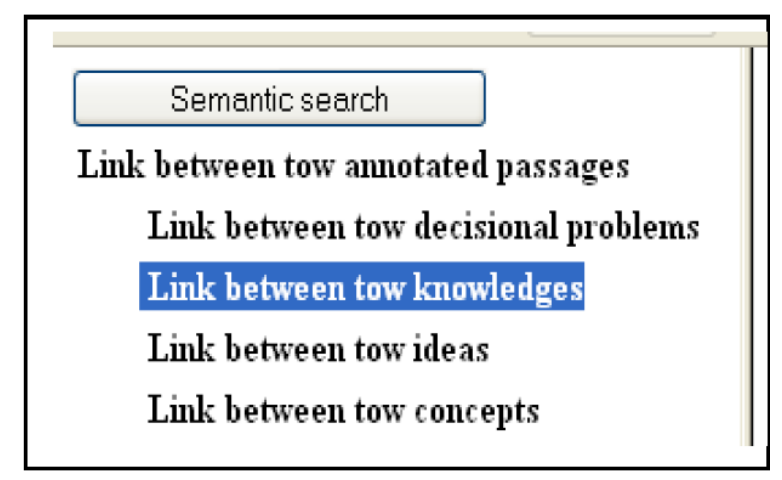

\section{Conclusions}

In this paper, we proposed a collective memory based on model semantic annotation for EI context. We used the interactions between EI actors in the form of annotation semantic in order to collect them and memorise them in a collective memory. In particular, our tool aims to facilitate the communication, the sharing of the comprehension of problem decisional, exploiting the collective intelligence between EI actors for collaborative problem solving.

We model this shared language using ontologies. We propose then an ontology-based annotation model. This model enables EI actors to describe their annotation's objectives using the two levels of semantics (domain and communication). Using this model, EI actors can communicate, memorise, re-use and retrieve their annotations easily guided by the ontologies. In the future, we will try to improve the tool annotation system with other concepts used in the EI context, and to validate our proposition by testing it with large domains.

\section{References}

Ardakani, M.K. and Oloonabadi, S.S.A. (2011) 'Collective memory as an efficient agent in sustainable urban conservation', in Secondini, P. et al. (Eds.): 2011 International Conference on Green Buildings and Sustainable Cities, Procedia Engineering, Vol. 21, pp.985-988.

Azouaou, F. and Desmoulins, C. (2006) 'Teachers' document annotating: models for a digital memory tool', International Journal of Continuing Engineering Education and Life-Long Learning, Vol. 16, Nos. 1/2, pp.18-34. 
Baker, M.J. (2009) 'Argumentative interactions and the social construction of knowledge', Argumentation and Education: Theoretical Foundations and Practices, pp.127-144, Springer Verlag, Berlin.

Barber, C., Cross, J., Yang, F. and Smith, P. (2005) 'Supporting shared analysis for mobile investigator', International Workshop Annotation for Collaboration, Paris, France.

Boudebza, S., Berkani, L., Azouaou, F. and Nouali, O. (2013) 'Semantic annotations and context reasoning to enhance knowledge reuse in e-learning', in Amine, A. et al. (Eds.): Modeling Approaches and Algorithms for Advanced Computer Applications, Vol. 488 of SCI, pp.419-428, Springer International Publishing, Switzerland.

Cabanac, G., Chevalier, M., Ravat, F. and Teste, O. (2007) 'An annotation management system for multidimensional databases', in Song, I-Y., Eder, J. and Nguyen, T.M. (Eds.): DaWaK'07: Proceedings of the 9th International Conference on Data Warehousing and Knowledge Discovery, Vol. 4654 of LNCS, pp.89-98.

David, H.W., Derek, N.L. and Souren, P. (2005) 'Implementation of a group decision support system utilizing collective memory', Information \& Management, Vol. 42, No. 4, pp.591-605.

Deng, Z. and Luo, L. (2007) 'An exploratory discuss of new ways for competitive intelligence on Web 2.0', IFIP International Federation for Information Processing, Integration and Innovation Orient to E-Society, Vol. 2, pp.597-604, Springer, Boston.

Dixon, R.A., Fox, D.P., Trevithick, L. and Brudin, R. (1997) 'Exploring collaborative problem solving in adulthood', Journal of Adult Development, Vol. 4, No. 4, pp.195-208.

Li, X., Zhu, Z. and Pan, X. (2010) 'Knowledge cultivating for intelligent decision making in small \& middle businesses', Procedia Computer Science, Vol. 1, No. 1, pp.2479-2488.

Macario, C.G.N. and Medeiros, C.B. (2009) 'A framework for semantic annotation of geospatial data for agriculture', International Journal of Metadata, Semantics and Ontologies, Vol. 4, Nos. 1/2, pp.118-132.

Moscato, F., Di Martino, B., Venticinque, S. and Martone, A. (2009) 'A collaborative framework for the semantic annotation of documents and websites', Journal Int. J. of Web and Grid Services, Vol. 5, No. 1, pp.30-45.

Noy, N.F., Sintek, M., Decker, S., Crubézy, M., Fergeson, R.W. and Musen, M.A. (2001) 'Creating semantic web contents with protégé-2000', IEEE Intelligent Systems, Vol. 16, No. 2, pp.60-71.

Odumuyiwa, V. and David, A. (2008) 'Collaborative information retrieval among economic intelligence actors', The fourth International Conference on Collaboration Technologies (CollabTech 2008), , Wakayama, Japan, pp.21-26.

Oliveira, J.P.M., Loh, S., Wives, L.K., Scarinci, R.G., Musa, D., Silva, L. and Zambenedetti, C. (2004) 'Applying text mining on electronic messages for competitive intelligence', E-Commerce and Web Technologies, pp.277-286, Heidelberg, Springer-Verlag.

Olusoji, O., Bolanle, O. and Victor, O. (2010) 'Dynamic knowledge capitalization through annotation among economic intelligence actors in a collaborative environment', Veille stratégique et scientifique, France, Toulouse.

Paul, S., Hasman, W.D. and Ramamurthy, K. (2004) 'Collective memory support and cognitiveconflict group decision-making: an experimental investigation', Decision Support Systems, Vol. 36, No. 3, pp.261-281.

Perez-Gallardo, Y., Alor-Hernandez, G., Cortes-Robles, G. and Rodriguez-Gonzalez, A. (2013) 'Collective intelligence as mechanism of medical diagnosis: the iPixel approach', International Journal of Expert Systems with Applications, Vol. 40, No. 7, pp.2726-2737, Elsevier.

Philippe, K. (2009) 'Tracer, Annoter et Mémoriser: Trois Actions pour Asseoir la Collaboration-Confiance du Veilleur et du Décideur', Veille Stratégique Scientifique et Technologique VSST'2009, France. 
Said, A. and Bretones, D. (2009) 'Economic intelligence and knowledge management two complementary facets of the same issue', International Journal of Applied Decision Sciences, Vol. 2, No. 1, pp.1-26.

Thiery, O. and David, A. (2002) Modelling of the User: Strategic Information Systems and Economic Intelligence, Report ADELI (French Association for the Development of Software), French, pp.28-37 [online] http://www.adeli.org/document/284-147p28pdf (accessed March 2013).

Wahiba, B.K. and Nouha, M. (2011) 'Using ontology for resume annotation', International Journal of Metadata, Semantics and Ontologies, Vol. 6, Nos. 3/4, pp.166-174. 